\title{
Effect of Mir-299-3p on the biological function of lung adenocarcinoma cell H1299 through targeting PTPRD
}

\author{
Yuan YUAN ${ }^{1}$, Xiaoquan $\mathrm{CHEN}^{1}$, Bin YUAN ${ }^{1}$, Yuqin $\mathrm{HU}^{1 \star}$
}

\begin{abstract}
The paper aimed to investigate the effects of up-regulation and down-regulation of Mir-299-3p on the proliferation, migration, invasion and apoptosis of lung adenocarcinoma cell line H1299, and to preliminarily explore the mechanism of Mir-299-3p regulating the biological behavior of lung adenocarcinoma cells. Mir-299-3p inhibitor and Mir-299-3p mimics were transfected into lung adenocarcinoma cell line H1299, and the transfection efficiency was measured by fluorescence microscopy. The successfully transfected lung adenocarcinoma cells were detected of proliferation, migration, invasion, and apoptosis. Mir-299-3p inhibitor and Mir-299-3p mimics were used to down-regulate and up-regulate Mir-299-3p respectively. Western blot was employed to detect the expressions of STAT3, p-STAT3, caspase3, Bcl-2, Bax and MMP-9 proteins in lung adenocarcinoma cells. Small interfering RNA technology was applied to construct a cell line with low expression of PTPRD. WB was used to measure the transfection efficiency to determine whether the down-regulation of PTPRD can partially or completely reverse the proliferation, migration, invasion, and apoptosis of lung adenocarcinoma cells after the down-regulation of Mir-299-3p. It was also determined whether the down-regulation of PTPRD can partially or completely reverse the effect of Mir-299-3p down-regulation on the expressions of p-STAT3, MMP-9, Cleaved-caspase3, Bcl-2 and Bax proteins in lung adenocarcinoma cells.
\end{abstract}

Down-regulation of Mir-299-3p can inhibit the proliferation and invasion of lung adenocarcinoma cell H1299, promote cell apoptosis, inhibit the expression of p-STAT3 and MMP-9, promote the expressions of apoptosis-inducing proteins such as Bax and Cleaved-caspase3, and also inhibit the expression of apoptosis inhibitor protein Bcl-2. The up-regulation of Mir-299-3p worked in the opposite way.

PTPRD is the direct target gene of Mir-299-3p. Mir-299-3p may directly target PTPRD to regulate the expressions of p-STAT3, MMP-9, Cleaved-caspase3, Bcl-2 and Bax proteins to affect the biological behavior of lung adenocarcinoma cells.

Keywords: Mir-299-3p; PTPRD; proliferation; migration; invasion; apoptosis.

Practical Application: Mir-299-3p on the Biological Function of H1299 Cell.

\section{Introduction}

At present, the high incidence and high mortality of lung cancer have always threatened the health and lives of the people. Research institutions around the world have conducted epidemiological investigations into lung cancer. According to reports, lung cancer incidence and mortality rank first among all malignant tumors in men, and rank second in women. As we all know, the pathogenesis of lung cancer is very complicated, and the current research has not been able to clarify it. Therefore, further exploration of the mechanism of lung cancer occurrence and development will be of great significance (Bray et al., 2018; Ferlay et al., 2019; Sun et al., 2018).

Studies have shown that miRNAs play a vital role in the occurrence of tumors. A number of studies have confirmed that miRNA can suppress or promote cancer in tumors. Some miRNAs are up-regulated in one tumor and down-regulated in another, so the same miRNA can play different roles in different tumors. In short, a miRNA can play different roles in different tumors, with complex mechanism (Linden et al., 2020; Arbour \& Riely, 2019).
This study detected the expressions of Mir-299-3p and PTPRD in lung adenocarcinoma, the effect of Mir-299-3p on the biological behavior of lung adenocarcinoma cells and its possible mechanism, and preliminarily clarified the molecular mechanism of Mir-299-3p targeting PTPRD, in which the biological behavior of lung adenocarcinoma cells was affected. It is expected to provide a new theoretical basis for early diagnosis, targeted therapy and prognosis evaluation of lung adenocarcinoma.

\section{Methods}

\subsection{CCK8 cell proliferation experiment}

Pulmonary adenocarcinoma cells H1299 were obtained, and the cells were transfected with Mir-299-3p, Mir-299-3p inhibitor and $\mathrm{NC}$ respectively. After $6 \mathrm{~h}$ of transfection, the medium was replaced with 1640 complete medium. The CCK8 method was used to measure cell proliferation, and the absorbance of each well at $450 \mathrm{~nm}$ was measured with a microplate reader. 


\subsection{Cell scratch test}

Pulmonary adenocarcinoma cells H1299 were obtained and treated as above. Cell migration was observed at $0,12,24$, 48 , and $72 \mathrm{~h}$ time points.

\subsection{Cell migration and invasion experiments}

The lung adenocarcinoma cells H1299 were obtained, and treated the same as above. The cell migration and invasion were studied in the Transwell chamber.

\subsection{Flow cytometry experiment}

The lung adenocarcinoma cells H1299 were obtained and treated the same as above. The apoptosis of the cells was detected by flow cytometry.

\subsection{Western blot detection}

The lung adenocarcinoma cells H1299 were obtained and treated as above. The protein sample was extracted. After SDS-PAGE electrophoresis and membrane transfer, the expression of each protein was detected by immunoreaction.

The cellular proteins were extracted from the Mir-299-3p inhibitor, Mir-299-3p inhibitor + siPTPRD and NC groups respectively, and the expression of each protein was detected by Western blot.

\subsection{Statistical analysis}

SPSS20.0 was employed. The measurement data were expressed as mean \pm standard deviation. A paired $t$ test was used for quantitative data analysis. The qualitative data were analyzed by X2 test. Spearman correlation analysis was used for correlation analysis. When $\mathrm{p}<0.05$, the difference is considered to be statistically significant.

\section{Result}

\subsection{The effect of Mir-299-3p on the proliferation ability of H1299 cells}

The results showed that, the proliferation ability of H1299 cells in the Mir-299-3p mimics, Mir-299-3p inhibitor and NC groups had no significant difference at 0 and $24 \mathrm{~h}$ time points, but the cell proliferation ability of the Mir-299-3p mimics group was significantly higher than that of the NC group after $48 \mathrm{~h}$. The cell proliferation ability of the Mir-299-3p inhibitor group was significantly lower than that of the NC group, indicating that Mir-299-3p can promote the proliferation ability of lung adenocarcinoma cells, as shown in Figure 1.

\subsection{The effect of Mir-299-3p on the scratch healing ability of H1299 cells}

The results showed that, the wound healing area in H1299 cells of the Mir-299-3p mimics group was significantly larger than that of the NC group when the scratches were healed for $24 \mathrm{~h}$, while the cell wound healing area of the Mir-299-3p inhibitor group was significantly smaller than that of the NC group. All differences were statistically significant, suggesting that Mir-299-3p can promote the scratch healing ability of lung adenocarcinoma cells, as shown in Figure 2.

\subsection{The effect of Mir-299-3p on the migration and invasion ability of $\mathrm{H} 1299$ cells}

The results showed that, in H1299 cells, the number of migration and invasion cells in the Mir-299-3p mimics group was significantly higher than that in the NC group, while the

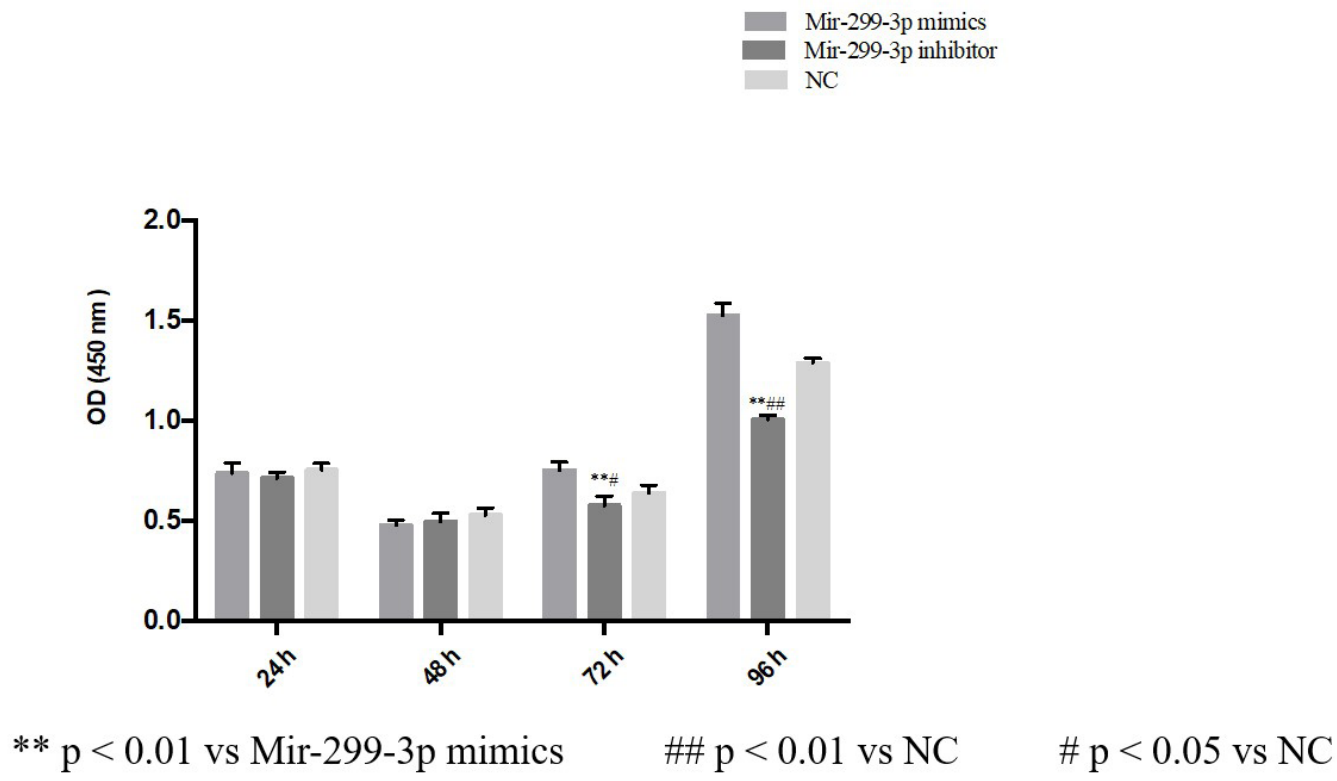

Figure 1. The effect of Mir-299-3p on the proliferation of H1299 cells. OD: Optical density. 


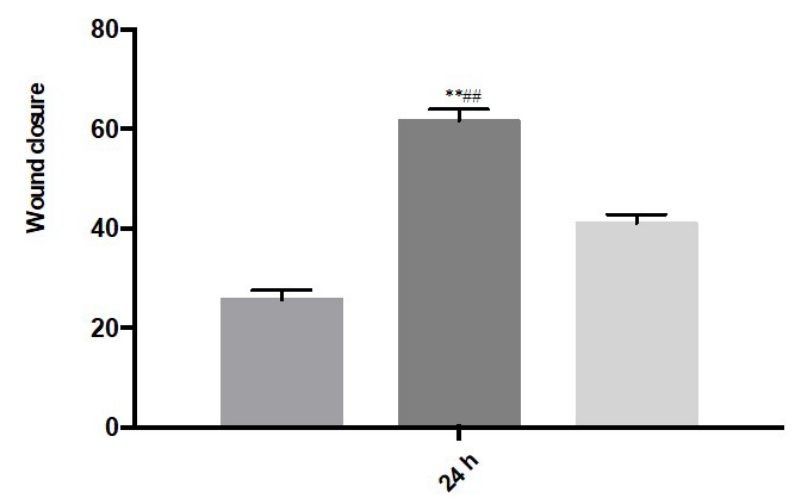

** $\mathrm{p}<0.01$ vs Mir-299-3p mimics \#\# $\mathrm{p}<0.01$ vs NC

Figure 2. The effect of Mir-299-3p on the scratch healing ability of H1299 cells.
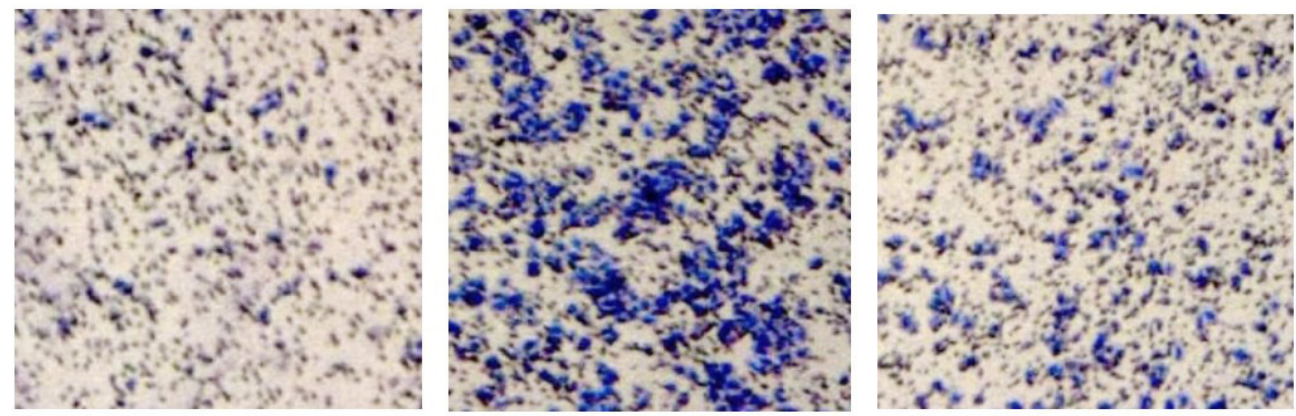

Migration
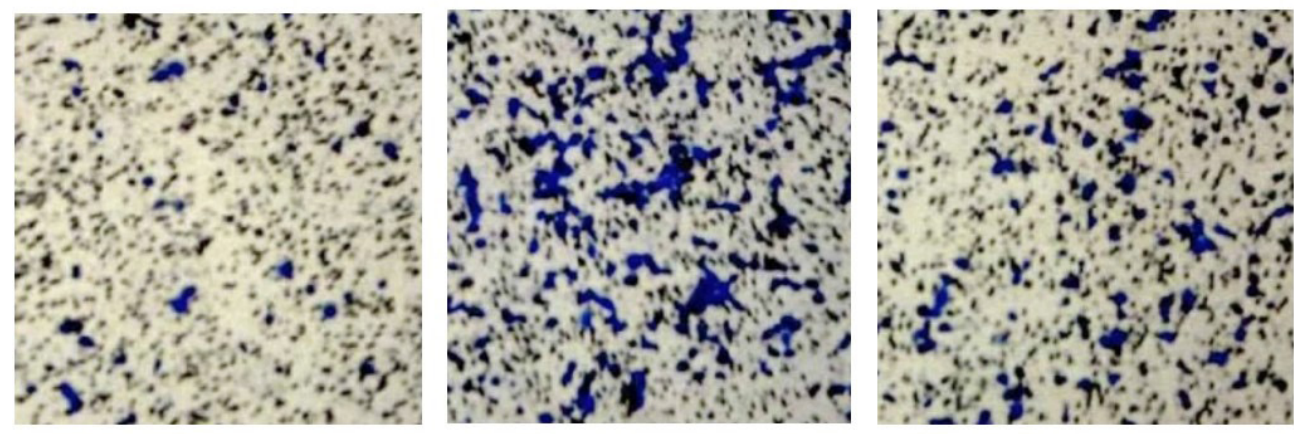

\section{Invasion}

Figure 3. The effect of Mir-299-3p on the migration and invasion ability of H1299 cells.

number in the Mir-299-3p inhibitor group was significantly lower than that in the NC group, as shown in Figure 3.

\subsection{The effect of Mir-299-3p on cell apoptosis}

The results showed that in $\mathrm{H} 1299$ cells, after $48 \mathrm{~h}$ of transfection, the proportion of apoptotic cells in the Mir-299-3p mimics group was significantly lower than that in the NC group, while the proportion of apoptotic cells in the Mir-299-3p inhibitor group was significantly higher than that in the NC group, indicating that Mir- 299-3p can inhibit the apoptosis of lung adenocarcinoma cells, as shown in Figure 4.

\subsection{The effect of Mir-299-3p on the expressions of various proteins}

The results showed that in H1299 cells, the up-regulation of Mir-299-3p can significantly promote the expression levels 

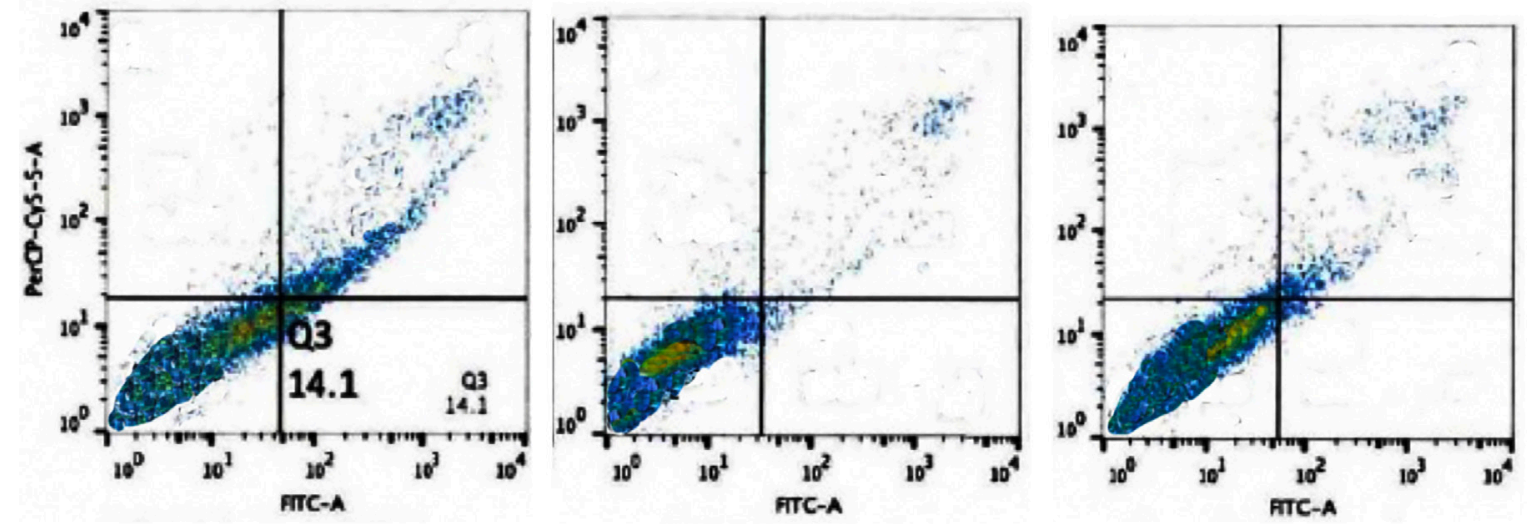

Figure 4. The effect of Mir-299-3p on cell apoptosis.

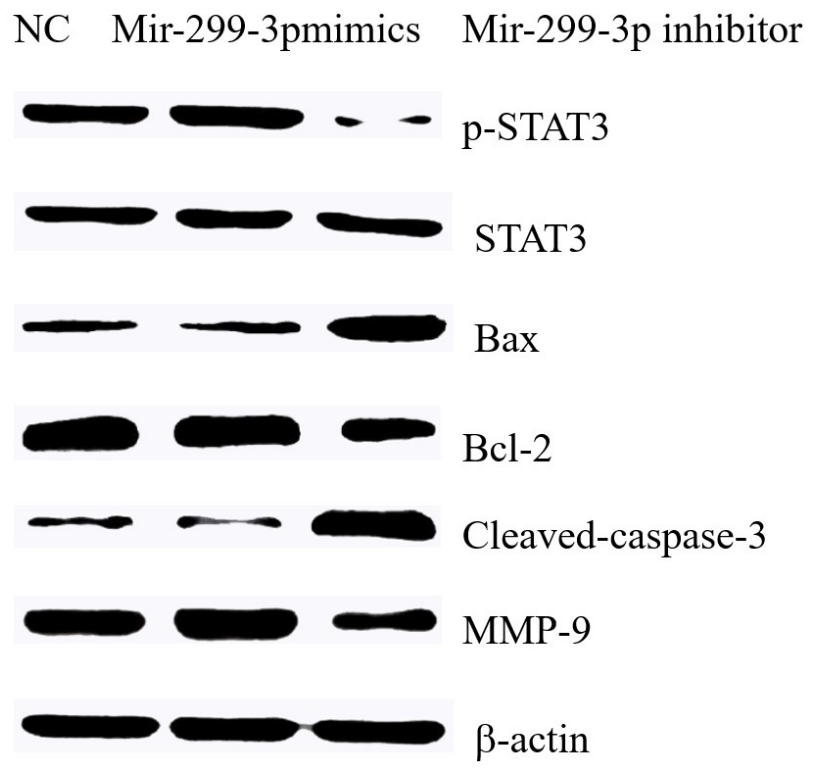

Figure 5. The effect of Mir-299-3p on the expressions of various proteins.

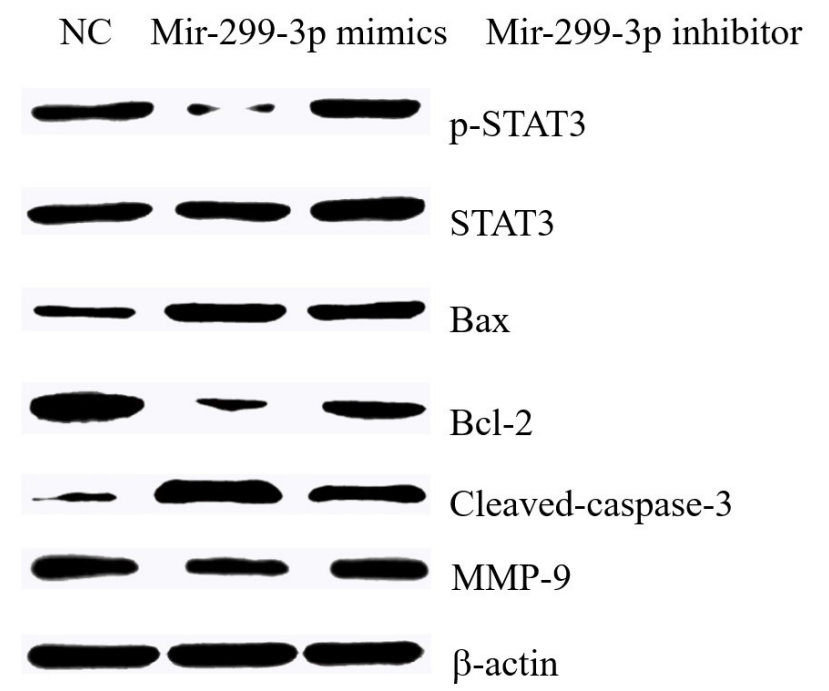

Figure 6. The effect of down-regulating Mir-299-3p and PTPRD on the expressions of various $\mathrm{H} 1299$ proteins. of P-STAT3 and MMP-9 proteins, and the down-regulation of Mir-299-3p can inhibit p-STAT3 Bcl-2 protein levels and promote the expressions of apoptosis induction proteins Bax and Cleaved-caspase3, as shown in Figure 5.

After down-regulating Mir-299-3p and then down-regulating PTPRD, p-STAT3 protein expression levels were restored, and the expression levels of Bax, Cleaved-caspase3, Bcl-2 and MMP-9 proteins were partially restored, as shown in Figure 6.

\section{Discussion}

The PTPRD gene is located on the chromosome, and the protein encoded by this gene is a member of the protein tyrosine phosphatase (PTP) family. PTP is a signaling molecule that regulates a variety of cellular processes, including cell growth, differentiation, mitotic cycle, and oncogenic transformation. PTPRD is one of the members of the Leukocyte Common Antigen (LAR) subfamily, which is composed of three members in human body, namely PTPRF, PTPRD and PTPRS. They are involved in a variety of human biological processes, such as embryonic development, cell growth inhibition, and axon guidance. Mutations in the LAR family are associated with developmental defects, such as cleft palate and various cancers, including breast, neck, lung, colon, and brain cancers. It has been found that PTPRD plays a key role in axon guidance, synapse formation, cell adhesion and tumor suppression (Liu et al., 2019a, b; Iacona et al., 2019).

At present, studies have confirmed that PTPRD plays a role in suppressing cancer carcinoma in a variety of tumors. It is found that down-regulating the expression of PTPRD can promote the proliferation, migration and invasion of gastric cancer cells. It has also been found that knocking down PTPRD in breast cancer cells can promote its clonal formation, cell migration and invasion capabilities, and can promote the growth of tumors in the body. In addition, PTPRD also increased the number of CD44/CD24-breast cancer stem cells and the expression of thousand-cell markers ALDH1 and OCT4. Besides, knocking down PTPRD can promote epithelial-mesenchymal transition of breast cancer cells. It has been published that overexpression of PTPRD can significantly inhibit the growth of head and neck squamous cell carcinoma. Furthermore, through the drug sensitivity test of 220 anticancer 
drugs on 45 head and neck squamous carcinoma cell lines, it was found that lower PTPRD expression cell lines are less sensitive to MEK inhibitors. It was also found that overexpression of PTPRD in acute myeloid leukemia cells can inhibit cell proliferation and clone formation, and induce cell apoptosis, while inhibiting the expression of PTPRD can promote cell proliferation. These effects may be related to the down-regulation of cyclinD1, c-myc and the up-regulation of Bax. After knocking down the expression of PTPRD, the migration ability of breast cancer cell MDA-MB-231 in vitro was significantly inhibited, and its lung colonization ability in mice was also significantly inhibited, suggesting that PTPRD plays a certain regulatory role in breast cancer cell metastasis (Gambale et al., 2019; Acun et al., 2015; Wu et al. 2019; Luo et al., 2018).

STAT3 belongs to a family of signal transduction and activation transcription factors. Research has found that STAT3 plays a carcinogenic effect in a variety of malignant tumors and is a recognized cancer-promoting gene. According to related reports, PTPRD is likely to exert a tumor suppressor effect by regulating the expression of STAT3. For example, after downregulating the expression of PTPRD, the phosphorylation of STAT3 protein will be inhibited. It was found in breast cancer that knocking down PTPRD can activate the STAT3 signaling pathway. The mechanism of PTPRD in tumors may be achieved by regulating the STAT3 signaling pathway. Overexpression of PTPRD in head and neck squamous cell carcinoma can activate the STAT3 signaling pathway. Deletion of PTPRD can lead to the activation of STAT3-mediated genetic programs. Loss of PTPRD can be determined as the cause of abnormal activation of STAT3 in gliomas. Knockdown of PTPRD in colon cancer cells can activate STAT3 and enhance its resistance to cetuximab, which can be reversed by inhibiting STAT3 (Qin et al., 2019; Kim et al., 2018; Bae et al., 2019; Ni et al., 2019; Wu et al., 2019; Feng et al., 2019).

\section{Conclusion}

In conclusion, PTPRD is the direct target gene of Mir-299-3p. Mir-299-3p may directly target PTPRD to regulate the expression of p-STAT3, MMP-9, Cleaved-caspase3, Bcl-2 and Bax proteins to affect the biological behavior of lung adenocarcinoma cells.

\section{References}

Acun, T., Demir, K., Oztas, E., Arango D., \& Yakicier, M. C. (2015). PTPRD is homozygously deleted and epigenetically downregulated in human hepatocellular carcinomas. OMICS: A Journal of Integrative Biology, 19(4), 220-229. http://dx.doi.org/10.1089/omi.2015.0010. PMid:25831062.

Arbour, K. C., \& Riely, G. J. (2019). Systemic therapy for locally advanced and metastatic non-small cell lung cancer: a review. Journal of the American Medical Association, 322(8), 764-774. http://dx.doi. org/10.1001/jama.2019.11058. PMid:31454018.

Bae, W. J., Ahn, J. M., Byeon, H. E., Kim, S., \& Lee, D. (2019). PTPRDinactivation-induced CXCL8 promotes angiogenesis and metastasis in gastric cancer and is inhibited by metformin. Journal of Experimental \& Clinical Cancer Research, 38(1), 484. http://dx.doi.org/10.1186/ s13046-019-1469-4. PMid:31805999.

Bray, F., Ferlay, J., Soerjomataram, I., Siegel, R. L., Torre, L. A., \& Jemal, A. (2018). Global cancer statistics 2018: GLOBOCAN estimates of incidence and mortality worldwide for 36 cancers in 185 countries.
CA: A Cancer Journal for Clinicians, 68(6), 394-424. http://dx.doi. org/10.3322/caac.21492. PMid:30207593.

Feng, R. M., Zong, Y. N., Cao, S. M., \& Xu, R. H. (2019). Current cancer situation in China: good or bad news from the 2018 Global Cancer Statistics. Cancer Communications, 39(1), 22. http://dx.doi. org/10.1186/s40880-019-0368-6. PMid:31030667.

Ferlay, J., Colombet, M., Soerjomataram, I., Mathers, C., Parkin, D. M., Piñeros, M., Znaor, A., \& Bray, F. (2019). Estimating the global cancer incidence and moxtality in 2018: GLOBOCAN sources and methods. International Journal of Cancer, 144(8), 1941-1953. http:// dx.doi.org/10.1002/ijc.31937. PMid:30350310.

Gambale, A., Russo, R., Andolfo, I., Quaglietta, L., De Rosa, G., Contestabile, V., De Martino, L., Genesio, R., Pignataro, P., Giglio, S., Capasso, M., Parasole, R., Pasini, B., \& Iolascon, A. (2019). Germline mutations and new copy number variants among 40 pediatric cancer patients suspected for genetic predisposition. Clinical Genetics, 96(4), 359-365. http://dx.doi.org/10.1111/cge.13600. PMid:31278746.

Iacona, J. R., Monteleone, N. J., Lemenze, A. D., Cornett, A. L., \& Lutz, C. S. (2019). Transcriptomic studies provide insights into the tumor suppressive role of miR-146a-5p in non-small cell lung cancer (NSCLC) cells. RNA Biology, 16(12), 1721-1732. http://dx.doi.org/ 10.1080/15476286.2019.1657351. PMid:31425002.

Kim, M., Morales, L. D., Jang, I. S., Cho, Y. Y., \& Kim, D. J. (2018). Protein tyrosine phosphatases as potential regulators of STAT3 signaling. International Journal of Molecular Sciences, 19(9), 2708. http://dx.doi.org/10.3390/ijms19092708. PMid:30208623.

Linden, S., Redig, J., Banos Hernaez, A., Nilsson, J., Bartels, D. B., \& Justo, N. (2020). Comorbidities and relevant outcomes, commonly associated with cancer, of patients newly diagnosed with advanced non-small cell lung cancer in Sweden. European Journal of Cancer Care, 29(1), e13171. PMid:31578054.

Liu, L., Wang, W., Gao, S., \& Wang, X. (2019a). MicroRNA208a directly targets Src kinase signaling inhibitor 1 to facilitate cell proliferation and invasion in non-small cell lung cancer. Molecular Medicine Reports, 20(4), 3140-3148. PMid:31432113.

Liu, S., Chen, J., Zhang, T., \& Chen, H. (2019b). MicroRNA-133 inhibits the growth and metastasis of the human lung cancer cells by targeting epidermal growth factor receptor. Journal of the Balkan Union of Oncology, 24(3), 929-935. PMid:31424644.

Luo, Y., Li, B., Zhang, G., He, Y., Bae, J. H., Hu, F., Cui, R., Liu, R., Wang, Z., \& Wang, L. (2018). Integrated Oncogenomic Profiling of Copy Numbers and Gene Expression in Lung Adenocarcinomas without EGFR mutations or ALK fusion. Journal of Cancer, 9(6), 1096-1105. http://dx.doi.org/10.7150/jca.23909. PMid:29581789.

Ni, K., Wang, D., Xu, H., Mei, F., Wu, C., Liu, Z., \& Zhou, B. (2019). MiR21 promotes non-small cell lung cancer cells growth by regulating fatty acid metabolism. Cancer Cell International, 19(1), 219. http:// dx.doi.org/10.1186/s12935-019-0941-8. PMid:31462892.

Qin, J. J., Yan, L., Zhang, J., \& Zhang, W. D. (2019). STAT3 as a potential therapeutic target in triple negative breast cancer: a systematic review. Journal of Experimental \& Clinical Cancer Research, 38(1), 195. http://dx.doi.org/10.1186/s13046-019-1206-z. PMid:31088482.

Sun, K. X., Zheng, R. S., Zeng, H. M., Zhang, S. W., Zou, X. N., Gu, X. Y., Xia, C. F., Yang, Z. X., Li, H., Chen, W. Q., \& He, J. (2018). The incidence and mortality of lung cancer in China. Zhonghua Zhong Liu Za Zhi, 40(11), 805-811. PMid:30481929.

Wu, L., Gao L., Kong, D., \& Xue, H. (2019). Loss of tyrosine phosphatase delta promotes gastric cancer progression via signal transducer and activator of transcription 3 pathways. Digestive Diseases and Sciences, 64(11), 3164-3172. http://dx.doi.org/10.1007/s10620-019-05637-z. PMid:31041642. 